\title{
Patient-Reported Experience of Diagnosis, Management, and Burden of Neuroendocrine Tumors
}

\author{
Results From a Large Patient Survey in the United States
}

\author{
Edward M. Wolin, MD, * John Leyden, MD, † Grace Goldstein, MA, $\ddagger$ Teodora Kolarova, MA, $\S$ \\ Ron Hollander, MS,// and Richard R.P. Warner, MD $\mid$
}

\begin{abstract}
Objectives: The aim of this survey was to examine the experience of patients with neuroendocrine tumors (NETs) to raise awareness of the NETrelated burden and identify unmet needs. Here, we report data from patients in the United States.

Methods: Patients with NETs participated in a 25-minute anonymous survey, conducted primarily online from February to May 2014. Survey questions captured information on sociodemographics, clinical characteristics, NET diagnostic experience, disease impact/management, interaction with medical teams, and NETs knowledge/awareness.

Results: Of 1928 patients who participated globally, the largest percentage was from the United States (39\%). Approximately $50 \%$ of US patients reported being diagnosed with other conditions before receiving their NET diagnosis, which for $34 \%$ took 5 years or more. Patients experienced many symptoms on a daily basis as a result of NETs, which had a substantial negative impact on their work and daily lives. Numerous improvements were suggested by patients, including better access to NET-specific treatments and medical teams/centers and better education for the management of disease-related and treatment-related symptoms.
\end{abstract}

Conclusions: This survey demonstrated the significant burden of NETs on patients' lives and identified key areas for improvement in diagnosis and long-term management, including better access to NET-specific treatments and specialist medical teams/centers.

Key Words: burden of illness, neuroendocrine tumors, patient-centered care, quality of life, surveys, rare diseases

From the *Neuroendocrine Program, Montefiore M-E Center for Cancer Care, Bronx, NY; †The Unicorn Foundation, Mosman, New South Wales, Australia; $\$$ The Carcinoid Cancer Foundation, Inc., White Plains, NY; §APOZ \& Friends, Sofia, Bulgaria; ||Neuroendocrine Tumor (NET) Research Foundation, Boston, MA; and 9 Mount Sinai School of Medicine, New York, NY.

Received for publication June 21, 2016; accepted December 15, 2016.

Address correspondence to: Edward M. Wolin, MD, Neuroendocrine Tumor

Program, Montefiore M-E Center for Cancer Care, 1521 Jarrett Pl, Bronx, NY 10461 (e-mail: ewolin@montefiore.org).

This study was supported by Novartis Pharmaceuticals Corporation.

Edward Wolin: honoraria and consultant/advisor: Novartis, Ipsen, Celgene, and Advanced Accelerator Applications; John Leyden: travel/accommodations/ expenses: The Unicorn Foundation Australia; Grace Goldstein: $\mathrm{COO}$ of The Carcinoid Cancer Foundation, Inc.; reports that the foundation has received grants from Novartis, Ipsen, Advanced Accelerator Applications, and Lexicon Pharmaceuticals; travel/accommodations/expenses: Novartis; Teodora Kolarova: travel/accommodations/expenses and honoraria: Novartis; reports that INCA has received grants from Novartis; Ron Hollander: executive director of the Neuroendocrine Tumor Research Foundation; Richard Warner: honoraria and consultant: Novartis, Pfizer, and Lexicon Pharmaceuticals; research funding: Lexicon Pharmaceuticals

Supplemental digital contents are available for this article. Direct URL citations appear in the printed text and are provided in the HTML and PDF versions of this article on the journal's Web site (www.pancreasjournal.com).

Copyright (C) 2017 The Author(s). Published by Wolters Kluwer Health, Inc. This is an open-access article distributed under the terms of the Creative Commons Attribution-Non Commercial-No Derivatives License 4.0 (CCBY-NC-ND), where it is permissible to download and share the work provided it is properly cited. The work cannot be changed in any way or used commercially without permission from the journal.

DOI: 10.1097/MPA.0000000000000818
(Pancreas 2017;46: 639-647)

$\mathrm{N}$ euroendocrine tumors (NETs) are a diverse group of malignancies that originate in neuroendocrine cells throughout the body, including the gastrointestinal tract, pancreas, and lungs. ${ }^{1}$ Neuroendocrine tumors are rare, although incidence rates have risen substantially in the United States (partly in response to improvements in diagnosis and increased awareness), with an ageadjusted incidence of 5.25/100,000 people in 2004. ${ }^{1}$ Neuroendocrine tumors can be clinically symptomatic because of the secretion of hormones (termed functional tumors) or silent/nonsecretory tumors, which are generally symptom free (nonfunctional). Lack of clear symptoms associated with NETs leads to delays in diagnosis, often until advanced stages of the disease when symptoms become more pronounced. ${ }^{2,3}$

Several types of medical specialists and health care professionals may be involved in the diagnosis and staging of NETs, requiring patients to undergo potentially repetitive and extensive examinations. The wide array of imaging techniques, biochemical testing, and expertise that are required to characterize the location and grade of the primary tumor and the extent of metastatic disease could possibly lead to patients receiving conflicting treatment plans and misdiagnoses. ${ }^{4,5}$ A large number of health care resources are also required for the long-term management of NETs, ${ }^{6,7}$ with qualitative studies highlighting the lack of a clear pathway of care for patients with NETs. ${ }^{8,9}$ Neuroendocrine tumor management should be highly individualized based on the burden and symptoms associated with the tumor and whether the aim of treatment is to ameliorate symptoms or prevent tumor growth. ${ }^{10}$ Difficulty in achieving an accurate diagnosis, limited access to NET-specific treatment centers or information about NETs, and inadequate support as part of ongoing care have been highlighted in previous studies as issues. 8,9 A multidisciplinary approach has been proposed to streamline the care of patients with NETs, involving the collaboration of diverse medical disciplines to optimize diagnosis, staging, and management, with the aim of enhancing clinical outcomes and improving patient satisfaction. ${ }^{4}$

The impact of NETs on reducing health-related quality of life (HRQOL) has been demonstrated using standardized testing. ${ }^{11-13}$ For the United States, Beaumont et $\mathrm{al}^{13}$ reported significantly worse HRQOL outcomes on all subscales of the 36-item Short Form Health Survey and 29-item Patient-Reported Outcome Measurement Information System in patients with NET compared with the general population. Furthermore, a qualitative study in Canadian patients with NET reported negative physical and psychological long-term effects, with many experiencing feelings of anxiety and struggling to cope with the chronic and unpredictable nature of their disease. ${ }^{9}$ A substantial financial burden has also been demonstrated in NET patients in the United States, 
particularly in the first year after receipt of the diagnosis and regardless of whether the patients received surgery or medical therapy. ${ }^{6}$ Although NETs have been shown to have a clear impact on patients' lives in North America, the patient perspective is not well documented, and the use of disease-specific quantitative surveys may provide further insight into the individual symptoms and concerns associated with NETs. ${ }^{13,14}$

The International Neuroendocrine Cancer Alliance (INCA) consists of 18 independent charitable organizations and patient groups from 15 countries, and aims to be the global advocate for patients with NETs. In collaboration with Novartis Pharmaceuticals Corporation, INCA conducted the first global patient survey to gather data about the experience of patients with NETs. This survey was designed to increase knowledge regarding NETs from a patient perspective. The main goals were to increase understanding of patient needs and challenges with regard to diagnosis, interactions with health care professionals, knowledge and awareness levels of NETs, information sources, and long-term management of the disease. Results of the global survey have been published elsewhere ${ }^{15}$; this publication presents data analyzed from patients in the United States with NETs. Results relevant to diagnosis and the impact that NETs have on patients' daily lives and the US health care system are highlighted.

\section{MATERIALS AND METHODS}

\section{Study Design and Participants}

Recruitment of patients was conducted primarily online via the use of Web site postings, e-mails, flyers, and social media channels of the INCA member organizations/patient advocacy groups. The global survey was conducted between February and May 2014.

\section{Survey Details}

The survey was designed and conducted as an equal collaboration between INCA and Novartis Pharmaceuticals. The survey domains and key questions were originally developed based on discussions at the roundtable INCA meeting in March 2013, held during the time of the 10th annual European Neuroendocrine Tumor Society conference in Barcelona, Spain. Hall \& Partners were commissioned to construct and professionally develop the questions; 14 NET patient health consumer groups within INCA had direct input into question development, and the final questionnaire was reviewed and edited by all members of INCA and Novartis between May and October 2013. For transparency, the Novartis logo was clearly displayed on all materials, and patients were notified that the INCA patient group partners or Novartis may use the data for disease awareness purposes. Hall \& Partners facilitated conduction of the survey from February to May 2014 and analyzed the results. This anonymous survey was designed to be predominantly completed online, with an estimated duration to completion of 25 minutes. For patients taking the survey in the United States, the survey was available in English and simplified Chinese. The English version of the survey used in the United States is included as Supplemental Digital Content 1, http:// links.lww.com/MPA/A587.

Sociodemographic information, including sex, age, employment status, and education level, was collected in addition to patient-reported clinical characteristics such as type, functional status, and grade of NETs. Furthermore, information on desired improvements, the burden of NETs on daily life and work, interactions with medical teams, and sources of information on NETs were also captured.
The survey was deliberately designed to be self-reporting to appropriately capture patient opinion. Question categories included (1) initial screening; (2) patient's current status; (3) diagnosis; (4) QOL; (5) NET management; (6) NET treatment; (7) NET education; and (8) demographics. The majority of survey questions were closed ended (ie, participants were provided options from which to choose). The only exceptions were open-ended questions related to the collection of numerical data (eg, age, number of visits per year to a NET specialist center, etc.). Patients were asked to rate certain parameters; responses included "not at all," "somewhat," "very," and "extremely" as graded descriptors, and when asked the degree to which they (dis)agreed with a particular statement, the descriptors "strongly" and "somewhat" were used.

\section{Data Analyses}

Data from patients in the United States were analyzed using the MERLIN (Merlinco, London, United Kingdom) survey software. Subgroup analyses were also performed based on the time since diagnosis $(<5$ or $\geq 5$ years $)$ and if the patient had visited a NET specialist center at least once a year. Responses were summarized using descriptive statistics, including percentages, means, and medians. Statistical significance is shown at a $95 \%$ confidence interval level $(P<0.05)$. Some responses are presented as a combination of the top 2 responses (eg, somewhat agree/strongly agree; a moderate amount/a lot).

\section{RESULTS}

\section{Patient-Reported Sociodemographics}

Participants with NETs from the United States represented $39 \%(758 / 1928)$ of the total number of patients in the global survey and were the largest number of respondents of any participating country. Patients were broadly distributed across the country, with 49 of 50 states represented (North Dakota was the only exception). The largest number of respondents were from California $(87 / 758 ; 11 \%)$, followed by Pennsylvania $(48 / 758 ; 6 \%)$, and Texas $(48 / 758 ; 6 \%)$. The average patient age was 57 years, with only $7 \%(52 / 758)$ of patients younger than 40 years, and the majority of respondents $(546 / 758 ; 72 \%)$ were women (Table 1). Patients were well-educated, with $51 \%$ (389/758) of patients having at least a bachelor's degree. At the time of the survey, $42 \%$ $(316 / 758)$ of patients stated that they were employed at least part time (including self-employment), 28\% (209/758) stated they were retired, and $20 \%(154 / 758)$ had a self-reported medical disability. A considerable number of patients required help with day-to-day activities; $59 \%$ (449/758) of patients had a close family member or friend who acted as a caregiver. At the time of the survey, the patients who participated had received their NET diagnosis a mean of 5.3 years ago.

\section{Patient-Reported Clinical Characteristics}

The most common site that patients reported for primary tumors was the gastrointestinal tract $(444 / 758 ; 59 \%)$, followed by the lung $(112 / 758 ; 15 \%)$, and pancreas $(111 / 758 ; 15 \%)$. The majority of patients reported having low-grade NETs: $35 \%(264 / 758)$ were grade 1 (low) and 17\% (127/758) were grade 2 (intermediate). However, 29\% (219/758) of patients reported that their physician did not give their NET a grade, and an additional $11 \%(82 / 758)$ stated that their physician did not give or discuss a grade for their NET. Almost half of patients $(363 / 758 ; 48 \%)$ had a functional NET, $33 \%(248 / 758)$ had a nonfunctional or asymptomatic NET, and $19 \%(147 / 758)$ said that their physician did not discuss the functional status of their NET with them or that they did not remember. 
Neuroendocrine tumors were reported by nearly three quarters of patients to have had at least a moderate negative impact on their lives. Thirty-nine percent (293/758) of patients felt that their NET had a large negative impact on their lives, 35\% (263/758) stated that they felt a moderate negative impact, and only $27 \%$ (201/758) of patients felt their NET had little-to-no negative impact on their lives. The most commonly reported negative impacts on patients' lives were overall energy levels $(540 / 758 ; 71 \%)$, finances (451/758; 59\%), and emotional health (441/758; 58\%) (Fig. 4A).

Patients reported making several lifestyle changes because of their NET. Just $6 \%$ (45/758) felt that they made no lifestyle changes as a result of their NET. Dietary changes $(487 / 758 ; 64 \%)$, increased spending on travel $(458 / 758 ; 60 \%)$, and cutting back on physical activities $(435 / 758 ; 57 \%)$ were the most commonly reported lifestyle changes indicated by patients in the survey.

Many patients also reported a negative impact on their work life (Fig. 4B). Only patients who were currently working (316/758; $42 \%$ of respondents) answered questions relating to their work life. Many patients $(196 / 316 ; 62 \%)$ stated that they needed to take days off work. Furthermore, 30\% (94/316) reported asking their employer to make accommodations. Other work impacts commonly conveyed by patients were working reduced hours $(65 / 316 ; 21 \%)$ and stopping work altogether for a time $(51 / 316$; $16 \%$ ). Patients who stated that they were not employed or able to work because of medical disability at the time of the survey $(192 / 758 ; 25 \%$ of total respondents) were asked if their NET was the reason they had to stop working, to which $79 \%$ (151/192) said yes. Patients who were retired at the time of the survey $(209 / 758 ; 28 \%$ of respondents) were asked about the previous impact NETs had on their work life. Thirty-one percent (65/209) stated they had to retire earlier than planned, $14 \%(29 / 209)$ had to take days off work, and $11 \%(22 / 209)$ had to stop working altogether for a time, whereas $22 \%(45 / 209)$ said their NET had not affected their work life.

\section{Management of NETs}

In the ongoing management of NETs, patients had an average of 3 health care professionals who were involved in their NET medical team. The health care professionals most commonly seen by patients were oncologists/hematologists $(659 / 758 ; 87 \%)$, general practitioners/primary care physicians $(400 / 758 ; 53 \%)$, and surgeons $(278 / 758 ; 37 \%)$. Patients who visited a specialist center at least once a year were more likely to see a wider range of specialists;
$43 \%(172 / 403)$ saw a surgeon and $40 \%(160 / 403)$ of patients saw a gastrointestinal specialist compared with $30 \%$ (106/355) and $26 \%(94 / 355)$ of nonvisiting patients, respectively. Those patients who reported having nurses $(204 / 758 ; 27 \%)$ and physician assistants $(114 / 758 ; 15 \%)$ as part of their medical team saw them the most frequently, at intervals of 1 and 2 months, respectively.

A total of 750 patients indicated that they had received a NET-related test; the vast majority of these patients $(511 / 750$; $68 \%$ ) reported having had up to 5 NET-related tests per year. However, $29 \%(220 / 750)$ of patients reported having tests 6 or more times a year; of these, $16 \%(118 / 750)$ had more than 10 . The most common tests performed on patients with NET were medical imaging tests $(715 / 758 ; 94 \%)$ (Table 2$)$

Long distances were traveled by NET patients to see their medical team. In total, $22 \%(168 / 758)$ of patients traveled more than 125 miles $(200 \mathrm{~km})$ to their medical provider; $23 \%(173 / 758)$ travelled $13-31$ miles $(21-50 \mathrm{~km})$, and $27 \%(204 / 758)$ of patients only had to travel up to 12 miles $(20 \mathrm{~km})$. The median distance traveled by all US NET patients in the survey was 30 miles $(48 \mathrm{~km})$. Patients who visited a specialist center at least once a year traveled more than twice the distance of nonvisiting patients, with a median of 50 miles $(80 \mathrm{~km})$ traveled for specialist center visitors compared with 20 miles $(32 \mathrm{~km})$ for nonvisiting patients.

\section{Perceptions of Care and Desired Improvements}

More than half of patients $(391 / 758 ; 52 \%)$ felt that the overall quality of NET treatments available today were good or very good; however, approximately a third of patients $(258 / 758 ; 34 \%)$ felt that NET treatments available today were poor or very poor. Patients who visited specialist centers felt significantly more satisfied with treatment than nonvisiting patients, with $57 \%$ (230/403) of patients rating treatment quality as good or very good compared with $45 \%(161 / 355)$ of nonvisiting patients.

Generally, patients felt that they did not have access to treatments of which they had heard. The most common treatments patients had heard of were surgery, chemotherapy, and nonchemotherapy-based drug therapies (Table 3 ). Nearly all $(718 / 758 ; 95 \%)$ patients had heard of surgery, $82 \%(618 / 758)$ said they had access to surgery, and $72 \%(546 / 758)$ said they had received or were receiving surgical treatment. Large differences were observed for chemotherapy and nonchemotherapy-based drug therapies in terms of treatments patients had heard of and treatments patients had access to: $80 \%(603 / 758)$ of patients had heard of chemotherapy,

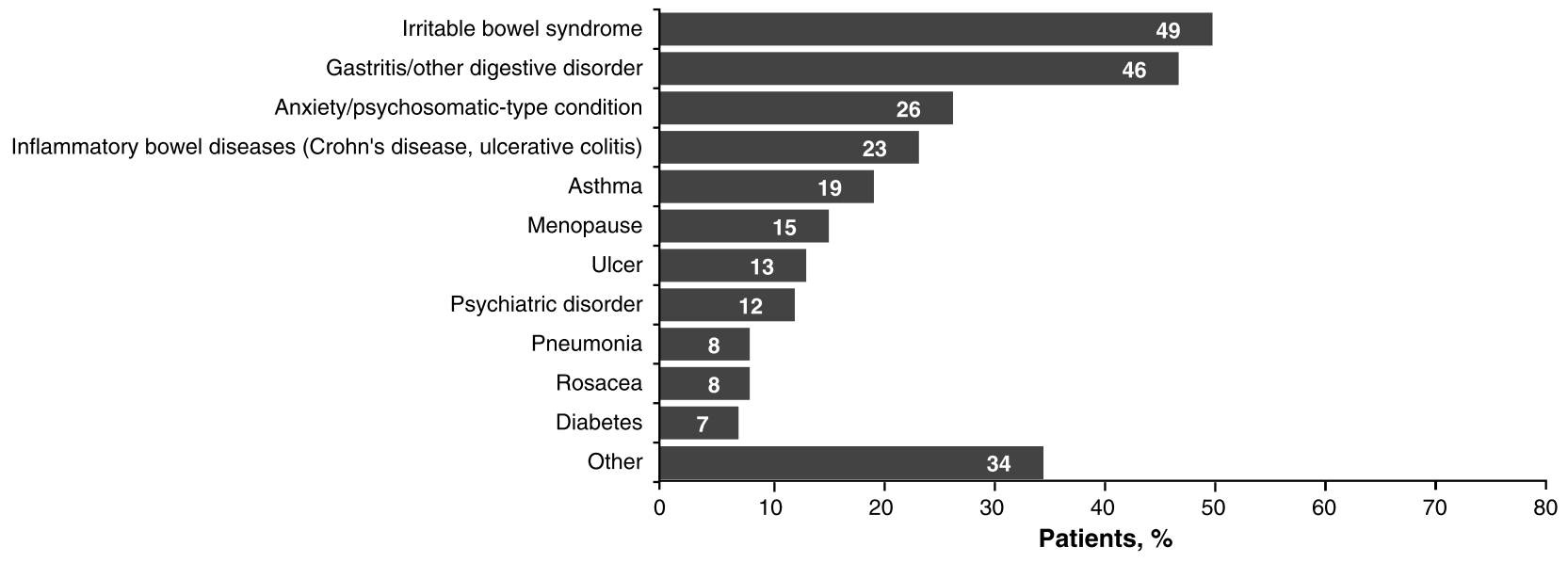

FIGURE 1. Diagnoses received before a NET diagnosis. Only includes conditions mentioned by more than $5 \%$ of patients.

Base: all US respondents who were diagnosed with other conditions $(n=368)$. Question: Which of the following conditions were you initially diagnosed with before receiving a NET diagnosis? 


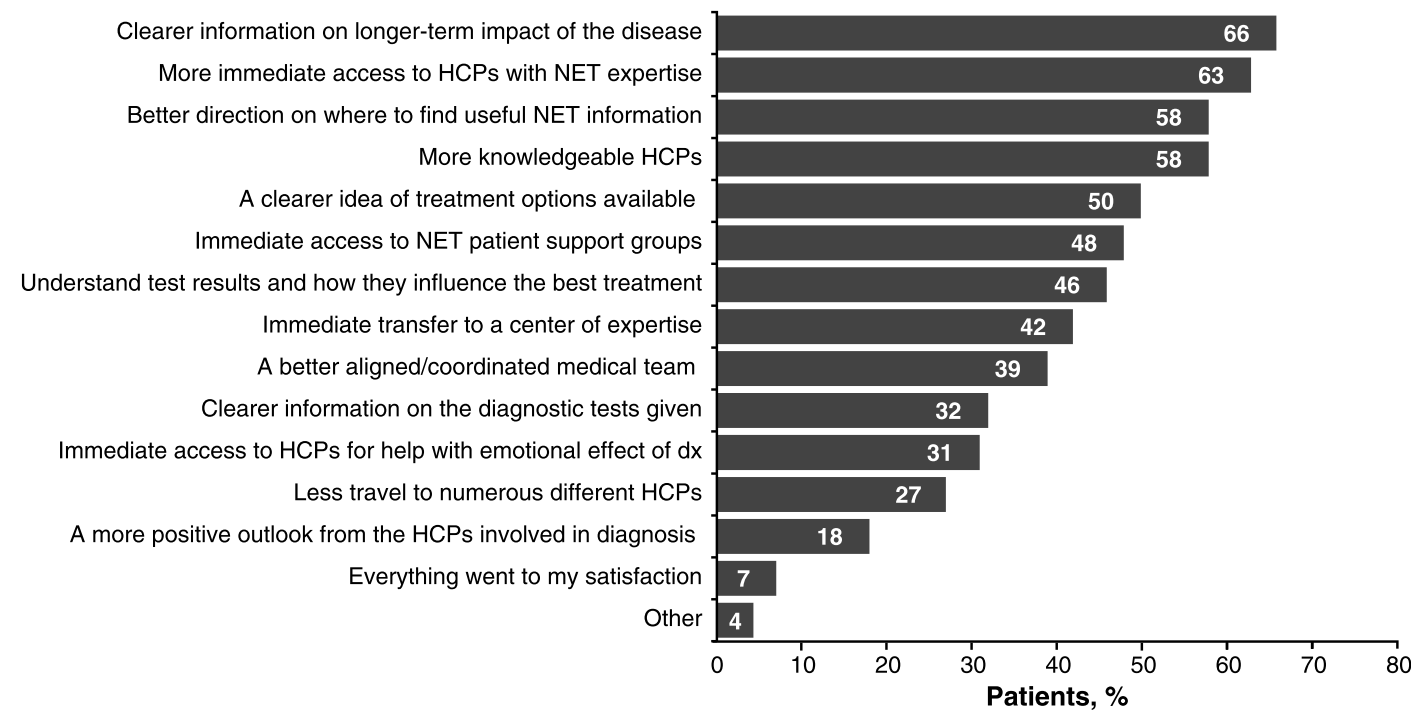

FIGURE 2. Desired improvements to the diagnosis of NETs. Base: all US respondents $(N=758)$. Question: Which of the following, if any, would have helped you have a better experience with your NET diagnosis? dx, diagnosis; HCPs, health care professionals.

but only $41 \%(310 / 758)$ said they had access to chemotherapy, and $22 \%(165 / 758)$ were currently receiving or had received chemotherapy. Although $83 \%(632 / 758)$ of patients had heard of nonchemotherapy-based drug therapies, only 58\% (442/758) said they had access to and $49 \%(371 / 758)$ said they were currently receiving or had received such therapies.

Overall, almost all patients $(688 / 758 ; 91 \%)$ felt that more treatment options were needed for the ongoing management of NETs. A majority of patients $(597 / 758 ; 79 \%)$ believed that there is room for improvement in the management of NETs. Eightythree percent (336/403) of patients who visit a NET specialist center at least once a year felt that they were very knowledgeable about the treatment options available to them, which was significantly higher than nonvisiting patients (243/355; 68\%). Overall, $76 \%$ (579/758) of patients felt they were very knowledgeable about treatment options.

Generally, patients felt that improvements could be made by health care professionals in terms of understanding and support. Most patients felt that health care professionals in general were either somewhat or not at all understanding (380/758; 50\%: 121/ $758 ; 16 \%)$ or supportive $(336 / 758 ; 44 \%: 58 / 758 ; 8 \%)$. Of those patients who reported seeing specific health care professionals, oncologists/hematologists $(492 / 659 ; 75 \%)$, nurses $(151 / 204$; $74 \%$ ), and surgeons $(192 / 278 ; 69 \%)$ were felt to be the most understanding health care professionals. The same health care professionals were considered to be the most supportive:

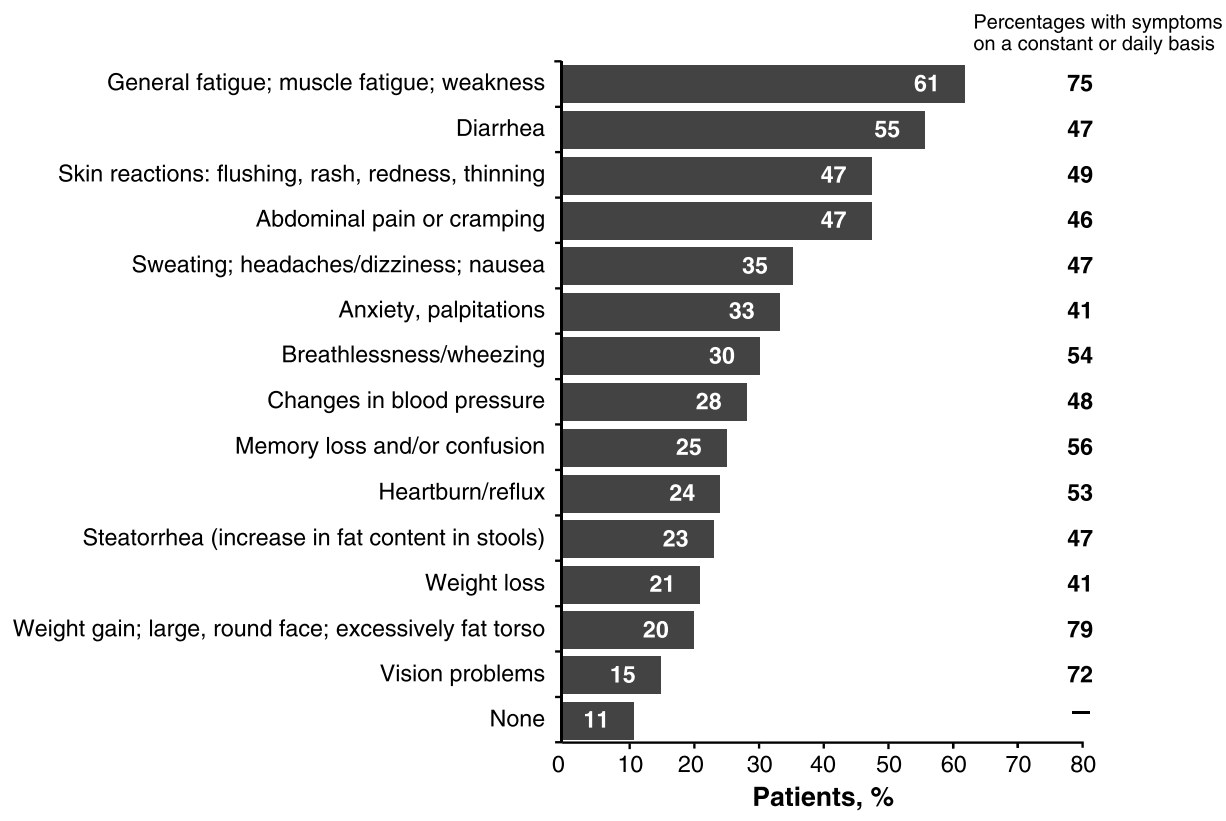

FIGURE 3. Symptoms experienced by patients as a result of NETs, often on a constant or daily basis. Within each subgroup of patients who experienced a particular symptom, the percentage that experienced that symptom on a constant or daily basis is provided. Base: all US respondents $(\mathrm{N}=758)$. Questions: Which of the following symptoms, if any, do you suffer from as a result of your NET? Select all that apply. How frequently do you suffer from each of the following symptoms as a result of your NET? 


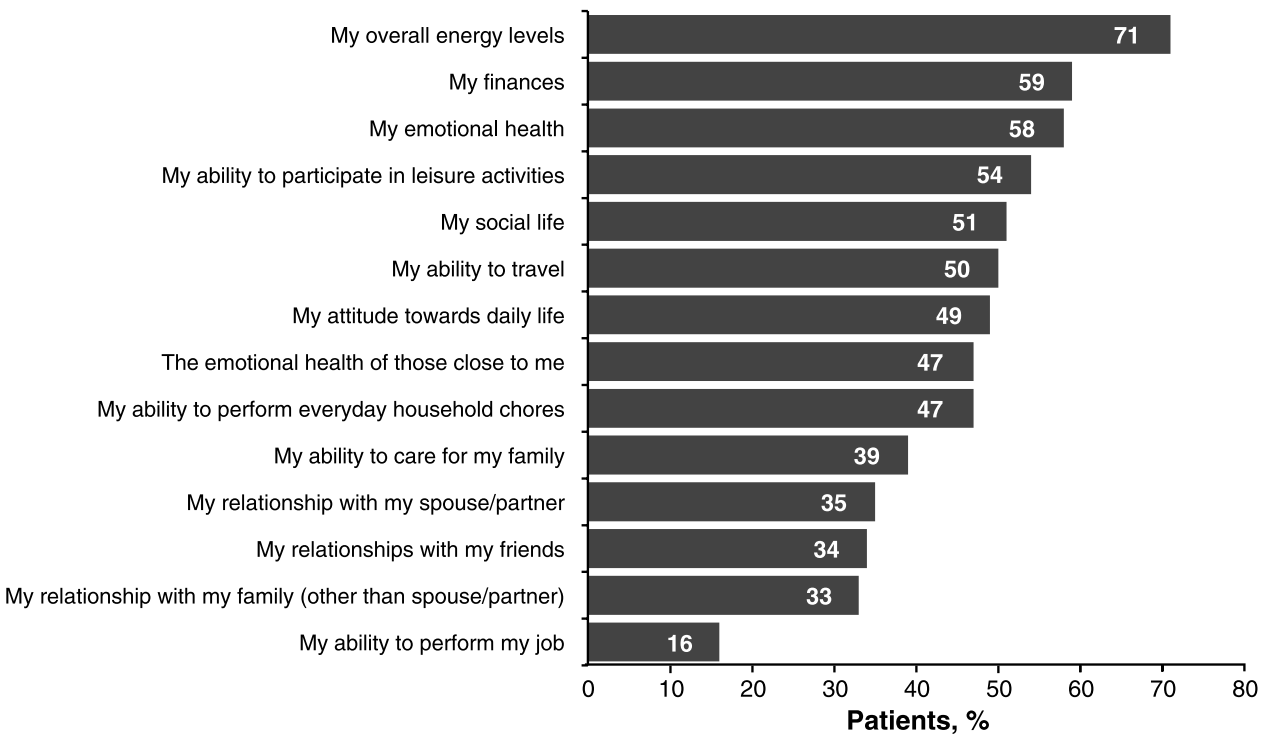

A

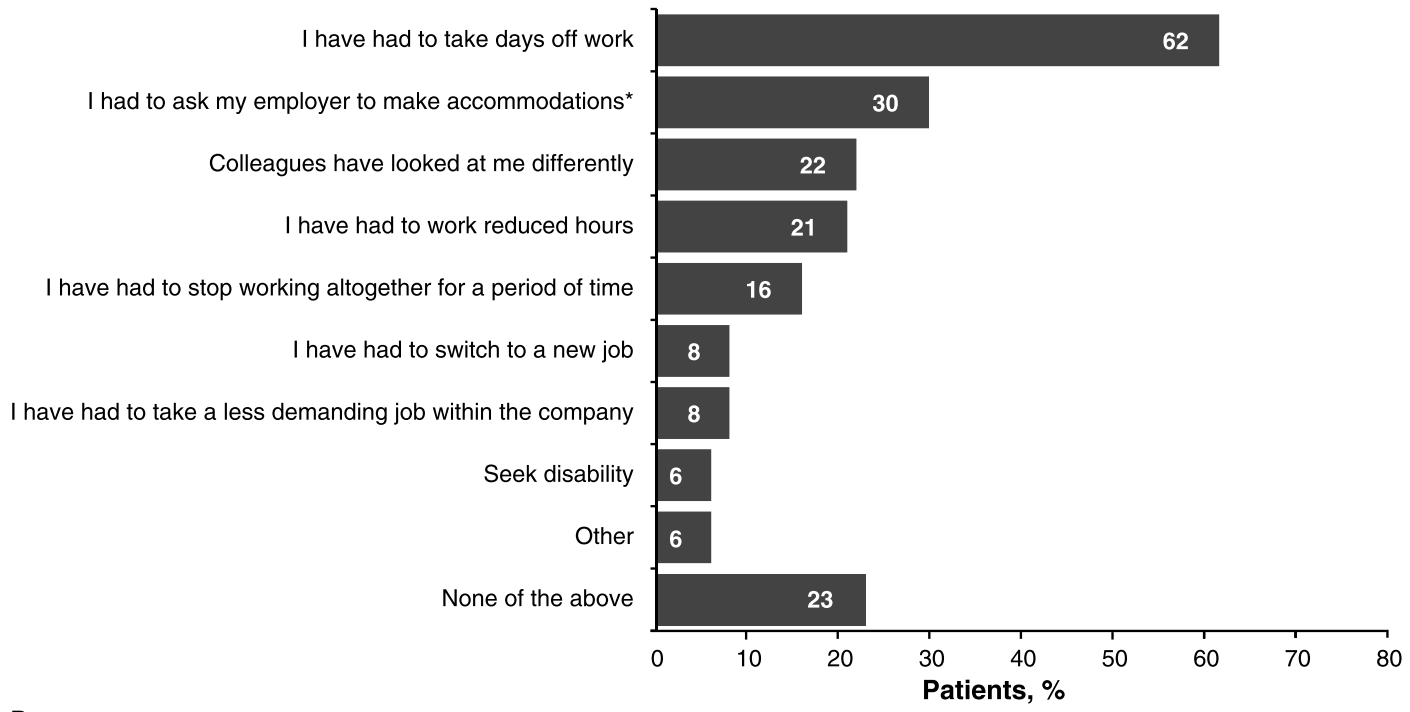

B

FIGURE 4. Negative impact of NETs on patients' daily lives (A) and work life (B). A, Base: all US respondents ( $N=758)$. Question: How much has each of the following areas of your life been negatively affected, if at all, by your NET? Top 2 responses shown (a moderate amount/a lot). B, *Accommodations include flexible work schedule, work from home, adaptive devices, opportunities for rest, etc. Base: US respondents who are working full time/part time or self-employed $(n=316)$. Question: Has your NET impacted you at work in any of the following ways? Please select all that apply.

$81 \%(225 / 278)$ of surgeons, $79 \%(162 / 204)$ of nurses, and $77 \%$ $(509 / 659)$ of oncologists/hematologists were viewed as extremely/ very supportive. However, only $57 \%$ (228/400) of patients viewed general practitioners/primary care physicians as extremely/very understanding, whereas $70 \%$ (278/400) viewed them as extremely/ very supportive.

The most frequently desired improvements for ongoing NET management were a wider range of treatment options (518/758; $68 \%)$, better access to NET experts $(471 / 758 ; 62 \%)$, and more treatments available "in my country that I see in other countries" (453/758; 60\%) (Fig. 5A). Significant differences were observed between specialist center visitors and nonvisitors. Patients who were diagnosed less than 5 years ago were more likely to want more information about participating in NET clinical trials than those diagnosed 5 or more years ago (54\% [235/438] vs 46\% [147/320]).
Patients believed that better access to NET-specific treatments and doctors would be beneficial in their NET care and help them to live better with NETs (Fig. 5B). Access to NET-specific medical treatments $(385 / 758 ; 51 \%)$ and NET medical teams $(344 / 758$; $45 \%$ ) were the most common responses by patients. Patients also reported wanting a better understanding of steps that could be taken to help manage disease-related $(336 / 758 ; 44 \%)$ or treatmentrelated symptoms $(304 / 758 ; 40 \%)$ and more awareness of the condition in general $(318 / 758 ; 42 \%)$. Patients who visited NET specialist centers felt they did not need as many improvements to NET care as nonvisitors.

\section{DISCUSSION}

The use of social media and other online sources allowed the recruitment of a large number of patients for this survey, despite 
TABLE 2. Tests Received for Ongoing NET Management

\begin{tabular}{lccc}
\hline & \multicolumn{2}{c}{ Patients Who Receive Each Test for Ongoing NET Management, \% } \\
\cline { 2 - 4 } & $\begin{array}{c}\text { All Patients } \\
(\mathbf{N}=\mathbf{7 5 8})\end{array}$ & $\begin{array}{c}\text { Patients Visiting Specialist } \\
\text { Centers at Least Once/Year } \\
(\mathbf{n = 4 0 3 )}\end{array}$ & $\begin{array}{c}\text { Patients Not Visiting Specialist } \\
\text { Centers at Least Once/Year } \\
(\mathbf{n}=\mathbf{3 5 5})\end{array}$ \\
\hline Imaging or enhanced imaging* & 94 & $97^{\dagger}$ & 91 \\
CgA & 79 & $84^{\dagger}$ & 73 \\
Urinary 5-HIAA & 72 & 74 & 68 \\
Other blood tests (eg, fasting serum glucose, & 67 & $75^{\dagger}$ & 59 \\
$\quad$ fasting gastrin, glucagon, VIP, somatostatin) & 62 & 64 & 59 \\
$\quad$ Endoscopic procedures (eg, gastroscopy, & & $42^{\dagger}$ & 34 \\
Selonoscopy, capsule endoscopy) & 38 & 1 & 1 \\
I am not sure what tests I had & 1 & 11 & 10 \\
Other/none of the above & 10 & & \\
\hline
\end{tabular}

Base: all US respondents $(\mathrm{N}=758)$.

Question: Which of the following tests, if any, have you received for the ongoing management of your NET?

${ }^{*} \mathrm{CT}$, MRI, PET, SRS (Octreoscan, PET/CT with ${ }^{68} \mathrm{Ga}$, F-DOPA scan, FDG PET/CT, MIBG scan).

$\dagger P<0.05$.

5-HIAA indicates 5-hydroxyindoleacetic acid; CgA, chromogranin A; CT, computed tomography; MRI, magnetic resonance imaging; PET, positron emission tomography; SRS, somatostatin receptor scintigraphy; VIP, vasoactive intestinal peptide.

the fact that NETs are a rare disease. Findings from this survey of patients with NETs in the United States provide valuable information on the experience of NETs from a patient perspective. There is a paucity of relevant disease-specific quantitative data regarding the NET patient experience and QOL. ${ }^{14}$ Although this NET patient survey is not a validated survey of HRQOL, reporting patient-centered data, in addition to targeting improvements in primary care and patient experience, is beneficial to health care systems, ${ }^{16}$ and these data are becoming increasingly important endpoints in oncology clinical trials.

A delay in NET diagnosis increases the probability of metastatic disease, ${ }^{10}$ which is associated with poor survival. ${ }^{1}$ Early and accurate diagnosis of NETs is therefore an essential element in improving patient outcomes. Although the diagnosis of NETs has improved in recent years, reflected in part by an increase in the
US incidence, ${ }^{1}$ the average time to NET diagnosis reported by a third of patients in this survey was more than 5 years, with more than half of the patients having metastatic disease at the time of diagnosis. This highlights the need for further improvements in this area. Multidisciplinary teams within an institution may create a more streamlined procedure and allow a consensus on NET diagnosis to be reached earlier. As this survey showed, patients attended doctor appointments on approximately 13 occasions and were seen by a mean of 6 different health care professionals (including all doctors, specialists, and nurses) before receiving their diagnosis. Effective multidisciplinary teams at specialist NET centers offer the best opportunity to improve outcomes for patients with NETs. ${ }^{4}$

This survey characterized the significant burden of NETs on patients' lives, with $73 \%$ of patients reporting that NETs had at

TABLE 3. Availability of NET Treatments

\begin{tabular}{lccc}
\hline & $\begin{array}{c}\text { Treatments I Have } \\
\text { Heard Of, \% (*) }\end{array}$ & $\begin{array}{c}\text { Treatments I Have } \\
\text { Access To, \% ( } \dagger\end{array}$ & $\begin{array}{c}\text { Treatments I Have Received/Am } \\
\text { Currently Receiving, \% (\$) }\end{array}$ \\
\hline Surgery & $95^{\dagger \ddagger}$ & $82^{\ddagger}$ & 72 \\
Chemotherapy & $80^{\dagger \dagger}$ & $41^{\ddagger}$ & 22 \\
Drug therapy other than chemotherapy & $83^{\dagger \dagger}$ & $58^{\ddagger}$ & 49 \\
PRRT & $78^{\dagger \ddagger}$ & $20^{\ddagger}$ & 10 \\
Interventional radiology & $74^{\dagger \ddagger}$ & $39^{\ddagger}$ & 21 \\
Observation & $86^{\dagger \ddagger}$ & $61^{\ddagger}$ & 52 \\
Other/none of the above & $14^{\dagger \ddagger}$ & 7 & 5
\end{tabular}

Superscripted symbols appearing next to a given value indicate significant differences $(P<0.05)$ between the patient group specified in that column compared with those specified in the other columns.

Base: all US respondents $(\mathrm{N}=758)$.

Question: This is a list of available NET treatments. Please select those you have heard of, those you have access to (meaning they are available to you), and those you have received/are currently receiving for the treatment of your NET.

$\S$ In the United States, PRRT is only available in clinical trials.

PRRT, peptide receptor radionuclide therapy. 
least a moderate negative impact on their life and only 5\% reporting no negative impact at all. The reduced QOL experienced by patients in this survey is supported by findings from standardized HRQOL testing in previous studies of patients from Europe and the United States. ${ }^{1-13}$ This disease-specific survey further elucidated this multidimensional concept of QOL, representing perceptions of health and well-being and how patients coped with their disease, demonstrating that several lifestyle changes were required, including making dietary changes and stopping or cutting back on physical activities. Patients also reported a negative impact on their work life, with many forced to reduce working hours, stop working altogether for a time, or retire early. This adds to the already considerable financial burden associated with surgical or medical therapy for NET management. ${ }^{6}$ A retrospective study reported that monthly total health care costs of NET patients in the United States who were treated with medical therapy increased from $\$ 5629.70$ in the 6 months preceding the diagnosis to $\$ 9093.30$ in the 12 months following (an increase of more than $60 \%$ ). For those receiving surgical therapy, the costs increased from $\$ 2547.90$ to $\$ 8810.40$ in the 6 months before and 12 months after the diagnosis, respectively. ${ }^{6}$

The results from this survey highlight several unmet needs by patients, including better access to NET-specific medical treatments and specialist NET centers, as well as access to a NET medical team. The diagnosis, management, and treatment of NETs is highly specialized, and health care professionals at a single institution will generally encounter a limited number of NET patients, making it difficult to maintain this specialized expertise. NET specialist centers have been proposed to facilitate the diagnostic process and reduce the burden on patients and health care systems. ${ }^{5,9}$ Overall, $53 \%$ of patients visited a NET specialist center at least once a year, which were located a median of 50 miles from a patient's residence, compared with 20 miles for nonspecialist centers. Many patients believed that access to NET specialist facilities and NET experts would be beneficial, and patients who visited NET centers felt that they received better care, which was reflected in the proportions of patients receiving specialized imaging and biochemical testing for NETs at these centers. Other

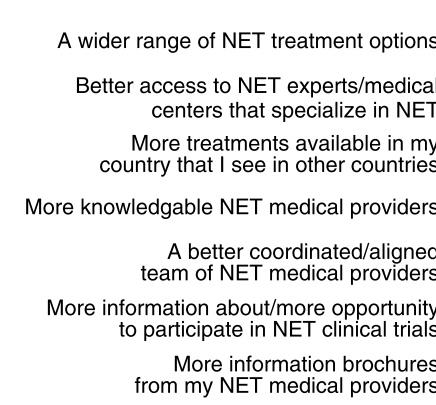

Other/none of the above

Oring

A

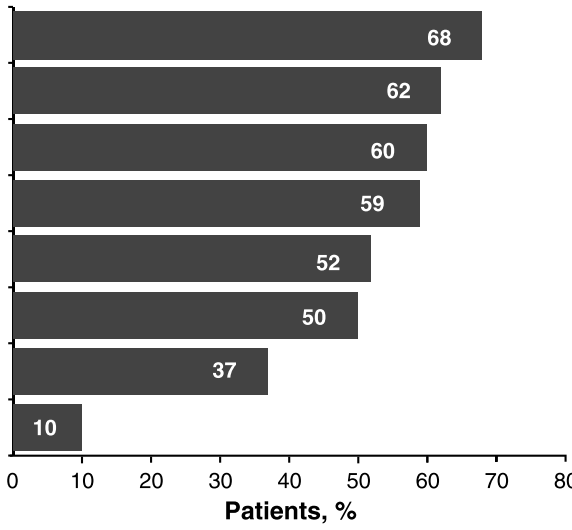

\begin{tabular}{|c|c|}
\hline $\begin{array}{l}\text { Visiting } \\
\text { specialist } \\
\text { center }\end{array}$ & $\begin{array}{l}\text { Not visitin } \\
\text { specialist } \\
\text { center }\end{array}$ \\
\hline 71 & 65 \\
\hline 56 & 69 \\
\hline 68 & 50 \\
\hline 53 & 66 \\
\hline 47 & 56 \\
\hline 48 & 53 \\
\hline 33 & 41 \\
\hline 10 & 9 \\
\hline
\end{tabular}

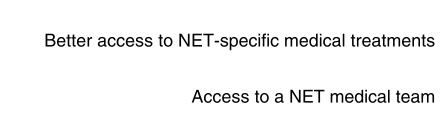

Better understanding of steps I can take to help manage my disease-related symptoms

More awareness of the condition in general to make it easier for me to be more open about it with other people

Better understanding of steps I can take to help manage my treatment-related symptoms

More knowledgable health team Materials that would help me better explain
my condition to family and friends Greater support to help me deal with the mental health consequences associated with the disease Greater understanding from my employer about the impact my NET has on me

Other/none of the above

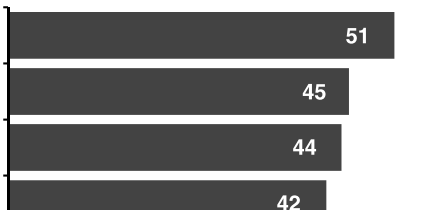

42

40

40

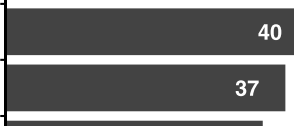

34

12

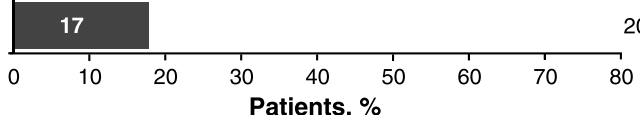

Visiting Not visiting specialist
center $\begin{gathered}\text { specialis } \\ \text { center }\end{gathered}$

$47 \quad 55$

$35 \quad 57$

$42 \quad 47$

$39 \quad 45$

$37 \quad 43$

$34 \quad 46$

$33 \quad 41$

$33 \quad 34$

$11 \quad 13$

$20 \quad 14$

B

FIGURE 5. Desired improvements in NET patient care. A, Bold numbers indicate statistical significance between patients visiting specialist centers versus those who do not. Base: All US respondents $(N=758)$. Question: Which of the following would help with the ongoing management of your NET? B, Bold numbers indicate statistical significance between patients visiting specialist centers versus those who do not. Base: all US respondents $(\mathrm{N}=758)$. Question: Which of the following, if any, would help you living with a NET? 
unmet needs identified by patients in this large survey were improving management of NETs, including education on disease and treatment-related symptom management, and improving awareness of the condition to assist in communicating with others.

Although the results from this survey are informative, it is important to note that the survey itself has several limitations. First, it did not use standardized and validated assessments of HRQOL. In addition, biased results could have been reported because recruitment for this survey was primarily online (Web sites, $41 \%$; social media, $32 \%$ ) or through patient advocacy groups (20\%), and a long time had passed since the initial NET diagnosis was made ( $42 \%$ were diagnosed $\geq 5$ years ago). The sociodemographics of the patients recruited were not fully representative of a heterogeneous NET population because respondents were more likely to be women ( $72 \%$ of respondents). Recruitment through advocacy groups might have enrolled more highly engaged, motivated care seekers who may have had a poorer prognosis. Moreover, details regarding experience at the time of diagnosis are more likely to be inaccurately recalled by those patients who had been diagnosed several years before participating in the survey.

Nevertheless, the results from this survey are informative and reflect the patient perspective. Validated patient-reported outcomes are becoming more prevalent endpoints in clinical studies because it is important to gain a true understanding of how patients experience their disease. Taken together, clinical outcomes and patient perspectives will aid in continuing to improve patient care and disease management. Although patient-reported surveys can be used to identify areas that require improvement as well as areas for further research, changes must be implemented by health care professionals for progress to be made.

This survey demonstrates the significant burden of NETs on patients' lives and has identified key areas for advancement in the diagnosis and long-term management of NETs. The use of NET specialist centers and health care professionals who specialize in NETs have been highlighted as areas in which patients would like to see further development in the future. Access to NET specialist facilities should lead to faster diagnoses and better management of NETs, which in turn should help to reduce the burden on both patients and health care systems.

\section{ACKNOWLEDGMENTS}

The authors thank the patients who participated in this survey and their patient advocacy partners in the United States who contributed to this project, The Carcinoid Cancer Foundation and NET Research Foundation. Hall \& Partners, a research organization, fielded the survey and analyzed the results.

This survey was funded by Novartis Pharmaceuticals Corporation and conducted as an equal collaboration between the International Neuroendocrine Cancer Alliance (INCA) and Novartis. Medical editorial writing assistance was provided by ApotheCom (Yardley, $\mathrm{Pa}$ ) and funded by Novartis.

\section{REFERENCES}

1. Yao JC, Hassan M, Phan A, et al. One hundred years after "carcinoid": epidemiology of and prognostic factors for neuroendocrine tumors in 35,825 cases in the United States. J Clin Oncol. 2008;26:3063-3072.

2. Vinik AI, Woltering EA, Warner RR, et al. NANETS consensus guidelines for the diagnosis of neuroendocrine tumor. Pancreas. 2010;39:713-734.

3. Boudreaux JP, Klimstra DS, Hassan MM, et al. The NANETS consensus guideline for the diagnosis and management of neuroendocrine tumors: well-differentiated neuroendocrine tumors of the jejunum, ileum, appendix, and cecum. Pancreas. 2010;39:753-766.

4. Metz DC, Choi J, Strosberg J, et al. A rationale for multidisciplinary care in treating neuroendocrine tumours. Curr Opin Endocrinol Diabetes Obes. 2012;19:306-313.

5. Modlin IM, Moss SF, Chung DC, et al. Priorities for improving the management of gastroenteropancreatic neuroendocrine tumors. $J$ Natl Cancer Inst. 2008;100:1282-1289.

6. Chuang CC, Bhurke S, Chen SY, et al. Clinical characteristics, treatment patterns, and economic burden in patients treated for neuroendocrine tumors in the United States: a retrospective cohort study. JMed Econ. 2015; 18:126-136.

7. Strosberg J, Casciano R, Stern L, et al. United States-based practice patterns and resource utilization in advanced neuroendocrine tumor treatment. World J Gastroenterol. 2013;19:2348-2354.

8. Griffiths J, Willard C, Burgess A, et al. Meeting the ongoing needs of survivors of rarer cancer. Eur J Oncol Nurs. 2007;11:434-441.

9. Feinberg Y, Law C, Singh S, et al. Patient experiences of having a neuroendocrine tumour: a qualitative study. Eur J Oncol Nurs. 2013;17: $541-545$.

10. Modlin IM, Oberg K, Chung DC, et al. Gastroenteropancreatic neuroendocrine tumours. Lancet Oncol. 2008;9:61-72.

11. Fröjd C, Larsson G, Lampic C, et al. Health related quality of life and psychosocial function among patients with carcinoid tumours. A longitudinal, prospective, and comparative study. Health Qual Life Outcomes. 2007;5:18

12. Haugland T, Vatn MH, Veenstra M, et al. Health related quality of life in patients with neuroendocrine tumors compared with the general Norwegian population. Qual Life Res. 2009;18:719-726.

13. Beaumont JL, Cella D, Phan AT, et al. Comparison of health-related quality of life in patients with neuroendocrine tumors with quality of life in the general US population. Pancreas. 2012;41:461-466.

14. Chau I, Casciano R, Willet J, et al. Quality of life, resource utilisation and health economics assessment in advanced neuroendocrine tumours: a systematic review. Eur J Cancer Care (Engl). 2013;22: 714-725.

15. Singh S, Granberg D, Wolin E, et al. Patient-reported burden of a neuroendocrine tumor (NET) diagnosis: results from the first global survey of patients with NETs. J Global Oncol. 2017;1:43-53.

16. Browne K, Roseman D, Shaller D, et al. Analysis \& commentary. Measuring patient experience as a strategy for improving primary care. Health Aff (Millwood). 2010;29:921-925. 